\title{
In-line Inspection of Pipelines by Using a Smart Pig (ITION) and Multivariate Statistical Analysis
}

\author{
MAGDA RUIZ, LUIS EDUARDO MUJICA, MARIO QUINTERO, \\ SERGIO QUINTERO and JOEL FLÓREZ
}

\begin{abstract}
The ferrous pipe structures of oil and gas production and, the transmission pipelines are, in majority, buried. Nowadays, phenomena like corrosion, mechanical stress, soil erosion, worker mistakes and damages caused by third parts have generated several problems over pipelines. Thus, major investment on integrity programs with In-Line Inspection Tools has been improved in order to examine the pipelines and avoid environmental, financial and social disasters.

Recently in Colombia, the Research Institute of Corrosion - CIC (Corporación para la Investigación de la Corrosión) runs their own smart pig ILI tool in pipelines. The inspection technology is based on inertial and operational trends, ITION (Inertial Technology Inspection and Operational Trends). Up to date, the technology has been tested several times inside of pipelines providing valuable information along of thousand kilometres. These records contain a huge amount of data that sometimes is difficult or impossible to understand by themselves.

A univariate statistical analysis can be used to determine the thresholds for each observationvariable. However, it does not analyse the correlated information between them. In this way, the main contribution of this work is the development of a methodology based on Principal Component Analysis (PCA) to monitor the structure by using the whole available variables gathered by ITION.
\end{abstract}

\section{INTRODUCTION}

The Oil \& Gas industry needs to know the status of their pipelines. Therefore identifying areas where pipe defects, deformations, corrosion products and other mechanical integrity problems appears is an important priority. The proper method to recognize the state of the entire pipe is the internal inspection (in-line inspection - ILI) by the movement of a vehicle instrumented with many sensors.

The Research Institute of Corrosion - CIC (Corporación para la Investigación de la Corrosión) developed an instrumented vehicle for inspection of critical losses of metal for pipes of carbon steel using a technique named Magnetic Flux Leakage MFL.

M. Ruiz and L.E. Mujica. Escola Universitària d'Enginyeria Técnica Industrial de Barcelona. Consorci Escola Industrial de Barcelona. Universitat Politècnica de Catalunya. BARCELONAtech. Dept. Applied Mathematics III. \{magda.ruiz , luis.eduardo.mujica\}@upc.edu.

M. Quintero, S. Quintero and J. Flórez. Department of Electronics, Research Institute of Corrosion CIC. Colombia. \{mquintero, squintero , jflorez\}@corrosion.uis.edu.co 
Reports with critical losses should allow operators to know the overall diagnosis of their pipes, and immediately consider the cases of higher probability of occurrence of a failure of the mechanical integrity.

However, these records contain a huge amount of data that sometimes is difficult or impossible to understand by themselves. A univariate statistical method can be used to determine the thresholds for each observation variable. Nonetheless, it does not analyse the correlated information between variables. In this way, the main contribution of this work is the development of a methodology based on Principal Component Analysis (PCA) [1] to monitor the structure by using the whole available variables by detecting statistically significant events or damages.

\section{REMANENT MAGNETIZATION FOR IN-LINE INSPECTION}

\section{Magnetic leakage technique (MFL)}

MFL is the most used technique for inspecting large pipelines, it uses permanent magnets which applies magnetic field to the pipelines. The magnetic field changes when exists damages (for instance metal-loss). The MFL technique demands a magnetic field strong sufficient to measure it. Besides the magnetic field should be uniform and consistent: uniform from inside out in order to spread out through the pipeline; consistent in magnitude, because during the monitoring process, it should be measurable at different locations. In deep, the magnetization process is carried out in the following way: when the applied field is removed, the flux density disappears and immediately after, the hysteresis effect appears. Next, the magnetic field is reapplied, the magnetization curve starts at an applied magnetic field equal to zero and a flux density equal to the remanent flux density [2]. In others words, when a magnetic field is imposed over a pipeline and next it disappears, some residual magnetization remains turns up [3]. Then a new hysteresis effect is generated.

The residual magnetism is a phenomenon in which the magnetic dipoles within the material are oriented in a particular value in the hysteresis curve of the material. Furthermore, when there are internal forces able to align the magnetic dipoles of a basic material, a permanent magnet is obtained. In a conductive material, magnetic fields that produce residual magnetism or magnetization can be induced [4]. The basic description of the hysteresis loop during magnetization and demagnetization of a typically ferromagnetic material can be seen in Figure 1. In the hysteresis curve it can be identified three zones in which their values of magnetizing force are used to inspect pipeline: MFL (Magnetic Flux Leakage), LFM (Low Frequency Magnetic) and RES (Residual Magnetic Field) [5].

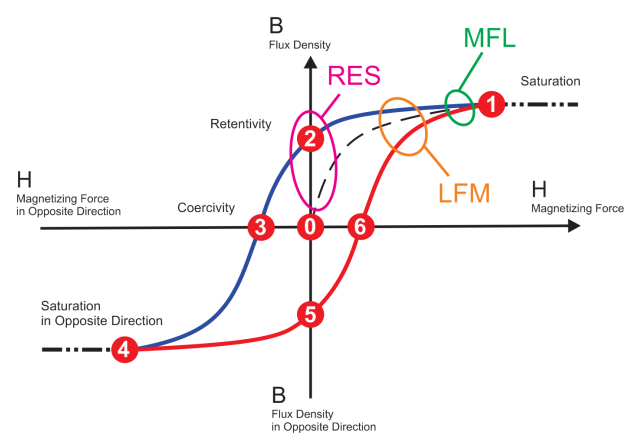

Figure 1. Typical hysteresis loop 


\section{ITION Technology}

The Corrosion Research Institute (CIC for its acronym in Spanish) is a technological development centre from Colombia. Its primary purpose is the production of knowledge, innovation and technology derived from daily industrial corrosion problems. As result, the CIC has developed the first smart Colombian pig In-Line Inspection tool (ITION) in pipelines (Figure 2).
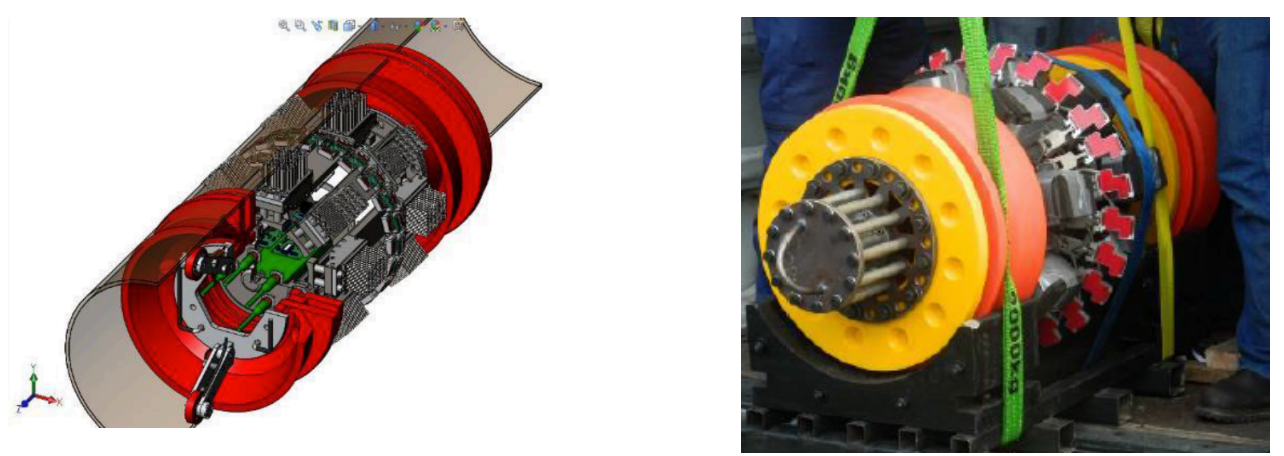

Figure 2: Inspection of Trends of Integrity and OperatioN-ITION- developed by CIC

The Technologies for Inspection of Trends of Integrity and OperatioN- ITION can often record and reconstruct the magnetic profile of the pipeline. This inspection tool consists of magnetic circuits, Hall effect sensors and electronics with the configuration and sufficient autonomy to record millimetre at the inner wall and the total length of pipelines. From the first time that the inspection is conducted, the current residual magnetic field is recognized. To report critical indications, ITION carries out studies on the trends of magnetic profile, pipe by pipe. Then, the monitoring of damages (critical metal losses) is performed by periodic comparison of changes in the magnetic profile and it is contrasted with damage reports previously known by the operator. To achieve the frequency of monitoring required by companies, ITION should be aware that the induced magnetic field intensity does not exceed the threshold for residual magnetization. Because, inducing fields may be subsequently reported as false positives.

The tool contains sensors that records information about acceleration, angular velocities, pressure and linear transducer that varies its output voltage in response to magnetic fields, providing a constant driving current to the sensors and amplifying the output signal. Besides, odometer systems measure the distance travelled by the tool allowing the calculation of the instantaneous speed. The flexibility and adaptation of the ITION technology allows its application using high frequency with low cost [6]. At the end, 17 sensors recording signals are plugged in the ITION'S technology as: intensity of axial movement; intensity rate of axial rotation; intensity resulting from the rotational; intensity remanent; remnant fields; propulsive force experienced by the tool, among others (all information about installed sensors is not provided by confidence reasons). The test is performed through in a pipeline for $24 \mathrm{Km}$ of length. This pipeline is made of sections of thin-walled steel tubing of $12 \mathrm{~m}$ length that are welded together using a circumferential weld. It has been in service for more than 15 years and nowadays it transport gas. A total of 14'449.944 measurements (samples) were collected by each sensor where the first 10 meters presents a high sampling frequency by calibration procedure (1'600.000 samples). 


\section{METHODOLOGY}

Structural changes cause changes in the magnetic field. The ITION technology creates a picture of the current condition of the structure. This objective is achieved by the periodic comparison of changes in the magnetic field profile with reports stored by the operator of the pipeline. Since it is not possible to give some diagnosis of the structure observing directly all measurements, PCA is applied to carry out a multivariable analysis, in other words, to analyse all measurements and its correlations as a whole [7].

The methodology that has been previously used by the authors for a multivariate analysis always include information related with the undamaged structure (baseline) to create a statistical model based on PCA. Afterwards, data collected by sensors when structure need to be assessed, are projected into the new space given by the PCA model [7][8][9][10]. In the current work, only one test with the ITION technology is available. This test has real data with 58 tags and damages across the pipeline. The pipeline owner's provides the locations and acronyms of these tags and damages (Figure 3). These tags include elements of the pipeline (e.g. VA belongs to valves) and damages, among others.

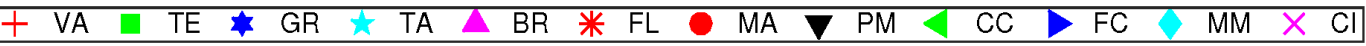

Figure 3. Tags provided by the owner of the pipeline

Considering some features of the raw data, these data are organized in three data matrices: Matrix 1 uses the whole dataset, where $n$ is number of experimental samples and, $m$ is the number of sensors in accordance with Equation $1\left(14^{\prime} 449.944 \times 17\right)$; In Matrix 2, samples from the first 10 meters are removed because of in this section a high sampling frequency is presented (11'434.944 × 17); Finally, in Matrix 3, the first 1'600.000 samples are removed because the odometer system stays at the same place $\left(12^{\prime} 849.944 \times 17\right)$.

$$
\mathbf{X}=\left(\begin{array}{cccccc}
x_{11} & x_{12} & \ldots & x_{1 j} & \ldots & x_{1 m} \\
\ldots & \ldots & \ldots & \ldots & \ldots & \ldots \\
x_{i 1} & x_{i 2} & \ldots & x_{i j} & \ldots & x_{i m} \\
\ldots & \ldots & \ldots & \ldots & \ldots & \ldots \\
x_{n 1} & x_{n 2} & \ldots & x_{n j} & \ldots & x_{n m}
\end{array}\right)=\left(v_{1}\left|v_{2}\right| \ldots\left|v_{j}\right| \ldots \mid v_{m}\right),
$$

where each row vector $\left(\boldsymbol{x}_{i}\right)$ represents measurements from all the sensors at a specific time instant. In the same way, each column vector $\left(v_{j}\right)$ represents measurements from one sensor (one variable) in the whole set of measurements.

Immediately after, the profile of the raw data is analysed. Some of these signals are depicted in Figure 4. For the purpose of this article, signals are processed without emphasis on the characteristics of the phenomenon that describes or the behaviour associated with any technique.

Next, the three statistical PCA models (in the same order than the data matrices X) are calculated (Transformation matrix or loadings denoted by $\mathbf{P}$ ) according to Equations 2 and 3. Equation 4 gives the data projected into the new three reduced spaces of the principal components. Besides for each one, statistical indices $Q$ and $T^{2}$ statistics are also determined (Equations 5 and 16). 
Signal 1

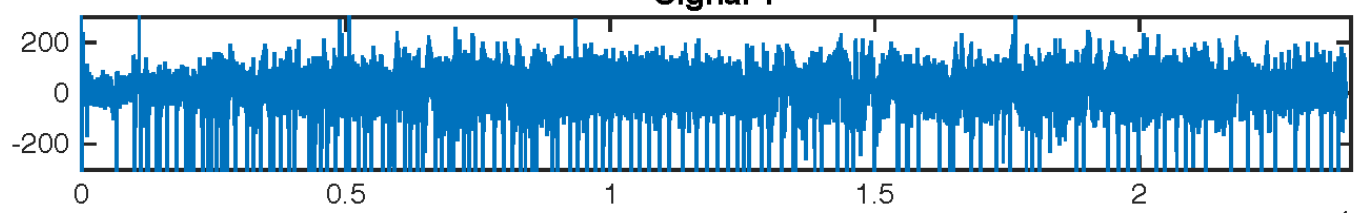

Signal 2
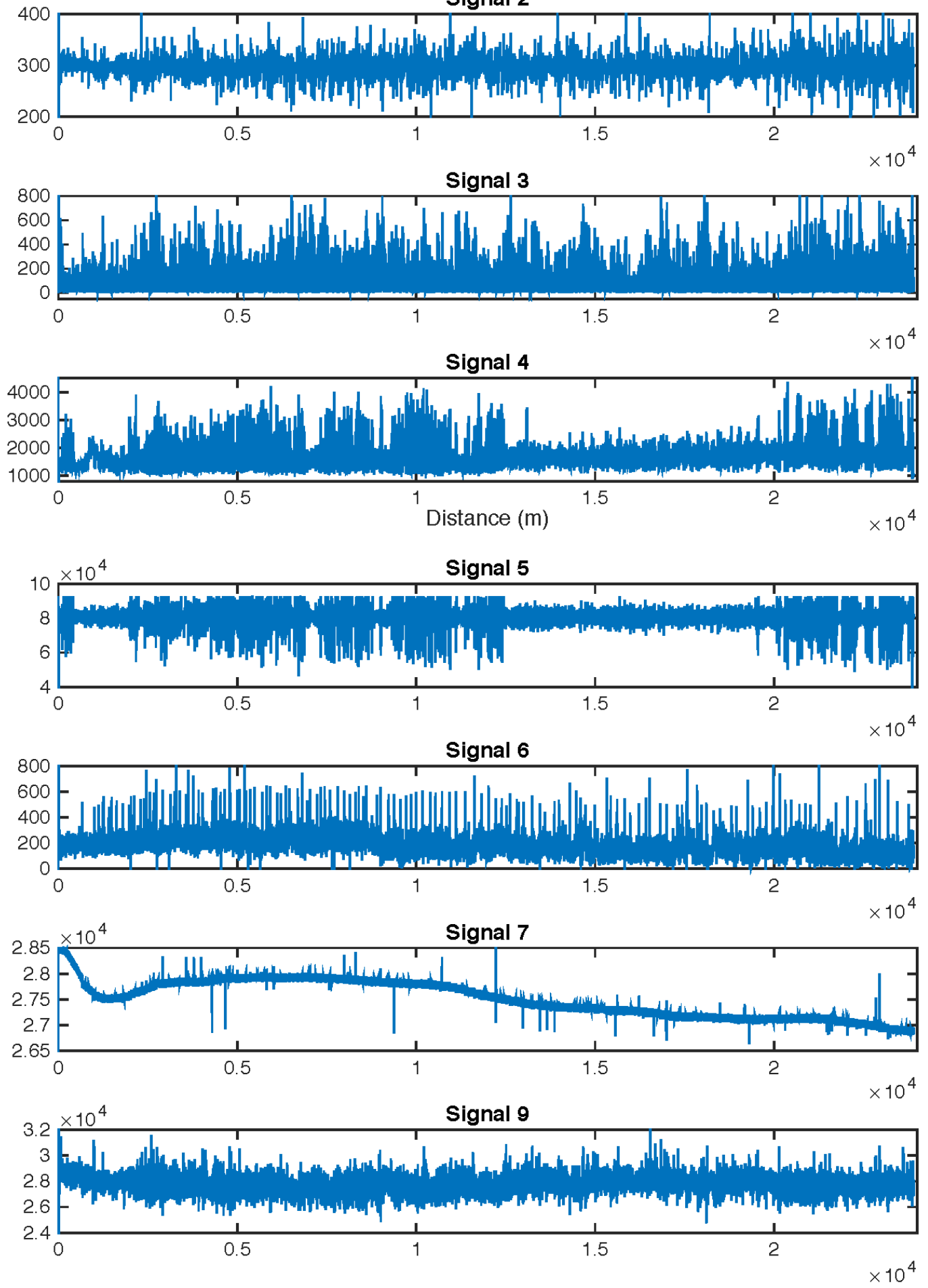

Figure 4. Some variable profiles of the original measurements detected by ITION 


$$
\begin{gathered}
\mathbf{C}_{\mathrm{X}} \equiv \frac{1}{n-1} \mathbf{X}^{\mathrm{T}} \mathbf{X} \\
\mathbf{C}_{\mathbf{X}} \mathbf{P}=\mathbf{P} \Lambda \\
\mathbf{T}=\mathbf{X} \mathbf{P} \\
Q_{i}=\tilde{\boldsymbol{x}}_{i} \tilde{\boldsymbol{x}}_{i}^{T}=\boldsymbol{x}_{i}\left(\mathbf{I}-\mathbf{P} \mathbf{P}^{\mathrm{T}}\right) \boldsymbol{x}_{i}^{T} \\
T_{i}^{2}=x_{i} \mathbf{P} \Lambda^{-1} \mathbf{P}^{\mathrm{T}} \mathrm{x}_{\mathrm{i}}^{\mathrm{T}}
\end{gathered}
$$

\section{RESULTS}

From Figure 4, we can see that these profiles did not yet any relevant information for damage detection and location. Since it is not possible to give some diagnosis of the structure observing directly the measurements, PCA is applied to carry out an analysis from the measurements and its correlations as a whole. Then, the three data matrices are scaled and the loading matrices, score matrices and indices $Q$ and $T^{2}$ are calculated. However, the results for detecting tags are not entirely satisfactory as is shown in figure 5. This Figure presents indices $Q$ and $T^{2}$ for Model 1 in which a big amount of possible false alarms are presented. Going back to the initial analysis, the profile of the signal 7, from the sensor temperature, should be considered. The temperature of the transported product is irrelevant for the goal of the analysis: tags detection and localization. Therefore, signal 7 is removed from the original data matrix and then the three PCA models are rebuilt.

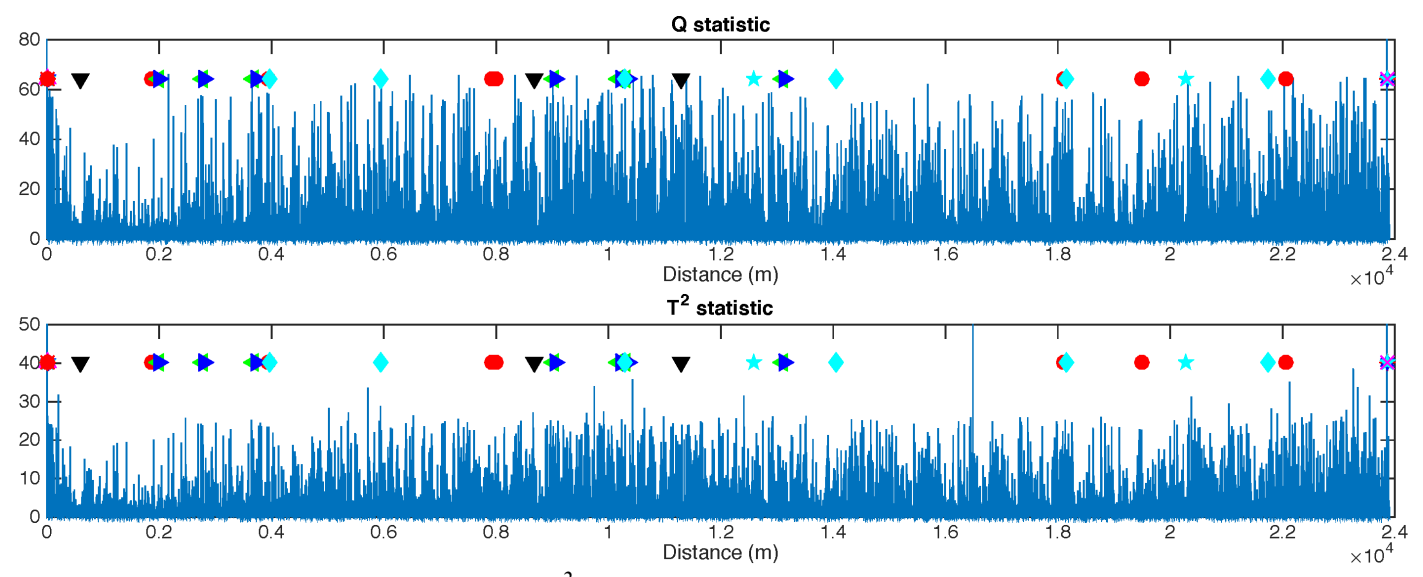

Figure 5. Indices $Q$ and $T^{2}$ for Model 1 including sensor 7 (Temperature)

Each new model is built with 4 principal components since around $90 \%$ of the cumulative variance is retained. Anew, scores and statistical indices $\left(Q\right.$ and $\left.T^{2}\right)$ are calculated. Figure 6 presents indices and the projections to score 1 until score 3 for Model 3 without temperature sensor (sensor 7). For this case, 44 tags should be detected and localized. According to Figure 6, results are improved. The three PM tags are detected. The sixth MA tag is weakly detected by index $T^{2}$, however by index $Q$, there is no doubt. Around the spot $0.2 \times 10^{4}$ meters $(2 \mathrm{Km})$, tags are remarked by $Q$ index. All scores and indices detect tags in $10 \mathrm{Km}$., however, in the interval from 14 $\mathrm{Km}$ to $18 \mathrm{Km}$, several maximum values are observed; therefore there exist false alarms. On the other hand, analysing more in deep the plots, it can be appreciated that for $Q, T^{2}$ and scores some values out of control appear every 12 meters (approximately) that could be attributed to the weld of the pipeline. 

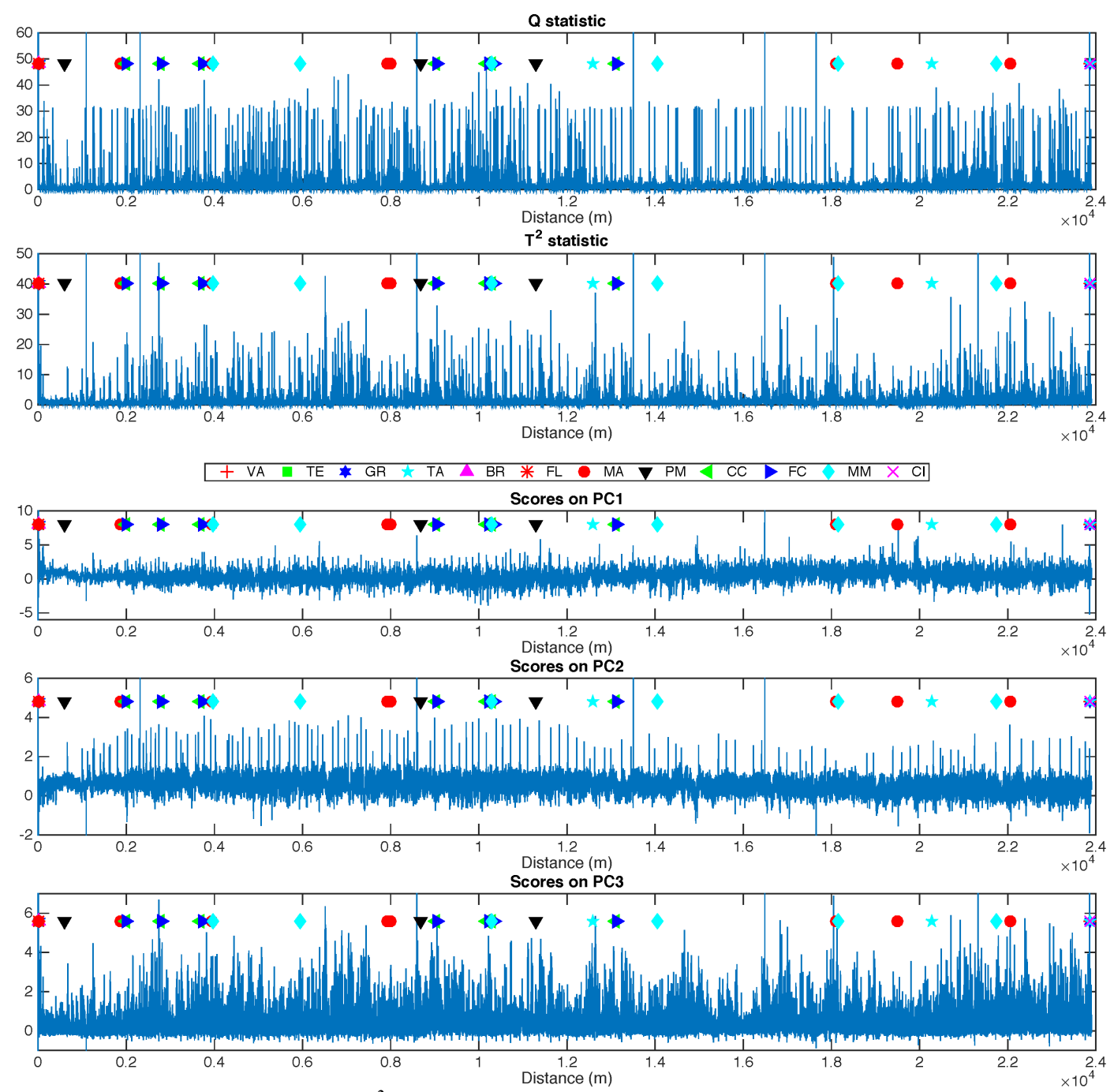

Figure 6. Indices $\left(Q\right.$ and $T^{2}$ ) and scores for Model 3, removing sensor 7 (Temperature)

\section{DISCUSSION AND CONCLUSIONS}

For continuously monitoring of pipelines to detect and locate damages, MFL technique is applied by means of comparisons of changes in the actual magnetic profile of the pipeline with reports previously known by the operator. Now, with the methodology based on PCA multivariable models, all sensors that are installed on the device are useful and their correlations are used to diagnose the state of the pipeline.

The smart pig tool (ITION) developed by CIC and the methodology (based on PCA) developed by UPC have been validated in a pipeline (currently in service) made of tubing sections welded every 12 meters.

The owner of the structure provided the location of 58 tags, these tags belong to different operational elements and damages of the pipeline, however any explanation of the meaning was given. A huge amount of measurements are gathered in the first run of the tool through $24 \mathrm{Km}$ of the pipeline. This information is processed by means of PCA and some indices are calculated for every location of measurement. The validation of the methodology is carried out by comparing the location of the "alarms" or values out of control of the mentioned indices and, the location of the tags. 
Results of the model 2, those that does not use data from the first 10 meters (because of oversampling), are not showed in this paper due to lack of space, but these are similar to the obtained using the model 3. In general, from results it is concluded that the localization of abnormal events (operational elements or damages) are improved considerably when redundant data are removed (e.g oversampling, smart pig without movement, etc). These results were considered as successful for the pipeline's owner and authors (despite that it is a novelty detection real application) due to the pipeline complexity. Even though several possible false alarms are presented, it is inferred that the pipeline welds could be the responsibilities.

On the other hand, the methodology must be improved if the owner is not interested in detecting welds. Besides a deep analysis of all variables is necessary to identify which variables are more influential to the different tags or phenomena existing along the pipeline (feature selection). Finally, it is important to emphasize that the computational cost is high due to the huge amount of data recorded by each sensor.

\section{ACKNOWLEDGMENT}

The authors thank to the "Escola Universitària d'Enginyeria Tècnica Industrial de Barcelona", "Consorci Escola Industrial de Barcelona", and "Ministerio de Ciencia e Innovación" in Spain through the coordinated research project DPI201128033- C03-01. Besides, to the Colombian Research Institute of Corrosion - CIC-.

\section{REFERENCES}

1. Jolliffe. I.T. (2002) Principal Component Analysis. Springer Series is Statistics. Springer-Verlag, second edition.

2. Nestleroth, J.B. and Davis, R.J. 1995. "The effects of remanent magnetization on magnetic flux leakage signals". Review of Progress in Quantitative Nondestructive Evaluation. 14: 483-490.

3. Zagidulin, R. V. and Muzhitskii V. F. 2003. "Estimation of the residual magnetic field strength inside a pipeline after testing by a magnetic flaw detector. Russian Journal of Nondestructive Testing, 39(7):549-552.

4. Babbar, V. and Clapham, L. 2003. "Residual magnetic flux leakage: a possible tool for studying pipeline defects", Journal of Nondestructive Evaluation, 22(4):117-125.

5. Seo, K., Han, J.M. and Park, G.W. 2007. "Effects of a remanent magnetization on the sensing signals in magnetic flux leakage type NDT". Electrical Machines and Systems, ICEMS. International Conference on. pp. 1932 - 1935

6. Perez I. and Dobmann G. 2013 "Surface Open Corrosive Wall Thinning Effects". International Workshop on Smart Materials, Structures \& SHM.

7. Mujica, L.E., Vehí, J., Ruiz, M., Verleysen, M,. Staszewski, W.J. and Worden, K. (2008) "Multivariate statistics process control for dimensionality reduction in structural assessment". Mechanical Systems and Signal Processing, 22:155-171.

8. Mujica, L.E., Rodellar, J., Fernández, A. and Güemes, A. (2010) "Q-statistic and T2-statistic PCAbased measures for damage assessment in structures”. Struc. Health Monitoring, 10(5):539-553.

9. Mujica, L.E., Ruiz, M., Pozo, F., Rodellar, J. and Güemes, A. (2014) "A structural damage detection indicator based on principal component analysis and statistical hypothesis testing". Smart materials and structures, 23(2):025014-1-025014-21.

10. Ruiz, M., Mujica, L.E., Berjaga, X. and Rodellar, J. (2013). "Partial least square projection to latent structures (PLS) regression to estimate impact localization in structures." Smart materials and structures, 22(2):025028. 